\title{
PROPOSTA DE UM FRAMEWORK PARA A PARTICIPAÇÃO EM UM EVENTO DA ENGENHARIA DE PRODUÇÃO A PARTIR DO MÉTODO TOPSIS
}

\author{
Kaique de Oliveira Dutton da Silva \\ Centro de Tecnologia da indústria Química e têxtil (SENAI CETIQT) \\ Rua Magalhães Castro 174 - Riachuelo - Rio de Janeiro - RJ - Brasil \\ kaiquedutton@gmail.com \\ Marcone Freitas dos Reis \\ Centro de Tecnologia da indústria Química e Têxtil (SENAI CETIQT) \\ Rua Magalhães Castro 174 - Riachuelo - Rio de Janeiro - RJ - Brasil \\ marconefreis11@gmail.com \\ Marcos dos Santos \\ Instituto Militar de Engenharia (IME) \\ Praça General Tibúrcio, 80 - Praia Vermelha - Urca - Rio de Janeiro - RJ \\ marcosdossantos_doutorado_uff@yahoo.com.br \\ Heider Silva Barros \\ Centro de Tecnologia da indústria Química e Têxtil (SENAI CETIQT) \\ Rua Magalhães Castro 174 - Riachuelo - Rio de Janeiro - RJ - Brasil \\ barrosheider72@gmail.com \\ Ernesto Rademaker Martins \\ Centro de Análise de Sistemas Navais (CASNAV) \\ Praça Barão de Ladário s/n - Ilha das Cobras - Rio de Janeiro - RJ - CEP: 20091-000 \\ radmart@yahoo.com.br
}

\begin{abstract}
RESUMO
Durante o decorrer do ano, ocorrem inúmeros eventos nacionais e internacionais reunindo muitos pesquisadores das mais diversas áreas e universidades com o simples propósito de realizar uma maior interação entre eles e o intercâmbio de ideias entre os participantes. No entanto, seja para um pesquisador ou para um estudante, eventualmente, não é fácil decidir em qual evento publicar a sua pesquisa, pois normalmente são procurados os eventos com maior destaque no mundo acadêmico. O objetivo desta pesquisa é selecionar o evento nacional mais apropriado para publicar um artigo na área de Engenharia de Produção, baseado nas opiniões de cinco pesquisadores na área. Para alcançar tal feito, foi utilizado um método multicritério de apoio à decisão, ou seja, foi utilizado o método TOPSIS (Technique for Order Preference by Similarity to Ideal Solution) a fim de ranquear os eventos e selecionar o mais apropriado. Com a utilização desta ferramenta, foi possível, através dos resultados das proximidades relativas, ranquear os eventos tendo como resultado o SBPO (Simpósio Brasileiro de Pesquisa Operacional) tendo com distância relativa 0,646964. A pesquisa alcançou os resultados desejados mediante o cenário proposto pelas opiniões dos profissionais da área.
\end{abstract}

Palavra-chave: Congressos; Método Multicritério; Apoio à Decisão; Método TOPSIS. 


\begin{abstract}
Throughout the year, there are numerous national and international events bringing together many researchers from various fields and universities for the simple purpose of engaging in greater interaction between them and the exchange of ideas among participants. However, whether it is for a researcher or student, it is sometimes not easy to decide which event to publish your research on, as the most prominent events in the academic world are usually sought. The objective of this research is to select the most appropriate national event to publish an article in the area of Production Engineering, based on the opinions of five researchers in the area. To achieve this, a multicriteria decision support method was used, in the TOPSIS (Technique for Order Preference by Similarity to Ideal Solution) method was used to rank the events and select the most appropriate. Using this tool, it was possible, through the relative proximity results, to rank the events resulting in the SBPO (Brazilian Operational Research Symposium) with a relative distance of 0.646964 . The research achieved the desired results through the scenario proposed by the opinions of professionals in the area.
\end{abstract}

Keywords: Congresses; Multicriteria Method; Decision Support; TOPSIS Method.

\title{
Como Citar:
}

SILVA, Kaique de Oliveira Dutton da; REIS, Marcone Freitas dos; SANTOS, Marcos dos; BARROS, Heider Silva; MARTINS, Ernesto Rademaker. Proposta de um framework para a participação de um evento da Engenharia de Produção a partir do método TOPSIS. In: SIMPÓSIO DE PESQUISA OPERACIONAL E LOGÍSTICA DA MARINHA, 19., 2019, Rio de Janeiro, RJ. Anais [...]. Rio de Janeiro: Centro de Análises de Sistemas Navais, 2019.

\section{INTRODUÇÃO}

Todos os anos ocorrem congressos científicos nacionais e internacionais das mais diversas áreas de pesquisa com o intuito de reunir o maior número de pesquisadores para realizar uma troca de ideias sobre as pesquisas desenvolvidas. Este tipo de evento pode agregar, não só os profissionais de cada área, mas também para os estudantes de graduação que queiram participar. No caso dos estudantes de graduação, a participação nestes congressos pode dar ao aluno uma oportunidade de obter novos conhecimentos em sua área de estudo além de conhecer os mais importantes pesquisadores dentro do universo acadêmico.

De alguns anos para cá, está se tornando cada vez mais comum os estudantes de graduação, junto a seus professores, submeterem artigos científicos para os congressos e simpósios que ocorrem no decorrer do ano. Esta prática não era tão comum em outros tempos, pois os alunos e professores não tinham essa relação de parceiros, porque nestes tempos os docentes entravam na sala de aula só para ministrar o conteúdo correspondente à disciplina da qual era encarregado. Para os graduandos, a prática de submeter trabalhos científicos para estes tipos de eventos é benéfica, pois isto pode contar no currículo do aluno, o que pode ajudá-lo profissionalmente ou na carreira acadêmica.

Contudo, muitas vezes os alunos dos cursos de graduação não têm muito acesso a estes tipos de eventos, pois na maioria das vezes eles ocorrem em cidades ou estados diferentes das universidades as quais os discentes estudam. Eventualmente, quando um aluno 
de alguma universidade ou faculdade, publica algum artigo nestes eventos, a própria instituição arca com o custo de transporte que o aluno vai ter para ir ao evento e apresentar o seu trabalho e os outros custos ficam por conta do próprio aluno.

Todos os congressos ou simpósios oferecem palestras e minicursos para os participantes, o que facilita a integração de todos os pesquisadores para a formação de um ambiente amistoso entre os participantes. Alguns eventos oferecem até coffee break e almoço para os participantes.

Como saber qual o melhor congresso a se submeter um artigo científico dentre os muitos que englobam a engenharia de produção? Este trabalho irá apresentar qual o melhor congresso dentre as alternativas que foram escolhidas pelos autores e irá apresentar uma solução baseada em suas opiniões.

\section{METODOLOGIA}

A presente pesquisa apresenta abordagens de natureza quantitativa e aplicada. Para a realização do referencial teórico do artigo, foram feitas pesquisas de trabalhos anteriores em anais de congressos da área de Engenharia de Produção e no portal de periódicos da CAPES (Coordenação de Aperfeiçoamento de Pessoal de Nível Superior). No que diz respeito ao estudo de caso, os valores dos critérios elencados como custo do evento, conveniência das datas, qualidade do site do evento e nível dos artigos foram pesquisados nos sites dos eventos. Quanto aos demais custos envolvidos como hospedagem e passagem, foram realizadas pesquisas na internet para se ter uma estimativa de quanto a mais o estudante de graduação terá que desembolsar. Quanto a importância do evento e a organização, foram realizadas entrevistas com cinco pesquisadores da área de Engenharia de Produção; e quanto a facilidade de acesso, foram feitas pesquisas na internet no que diz respeito as cidades onde ocorrem os congressos.

Como delimitação do estudo pode-se citar: a pesquisa restringiu-se apenas aos congressos nacionais, o aluno de graduação submeterá apenas um artigo para qualquer congresso elencado no estudo e o aluno terá uma participação no evento de três dias. Vale ressaltar que as pesquisas referentes aos custos envolvidos como passagem e hospedagem foram feitas no site decolar.com. É válido considerar que os custos, que serão mencionados no estudo de caso, serão custos aproximados.

Feito esses passos, foi utilizado o método TOPSIS (Technique for Order Preference by Similarity to Ideal Solution) com o objetivo de ranquear os eventos para a elucidação de qual seria o congresso mais apropriado para publicar um artigo na área de Engenharia de Produção, levando-se em consideração as opiniões dos profissionais que foram entrevistados.

\subsection{FUNDAMENTAÇÃO TEÓRICA}

\subsection{PESQUISA OPERACIONAL}

A pesquisa Operacional ou ainda PO surgiu na Inglaterra durante a Segunda Guerra Mundial para a solução de problemas de natureza logística, tática e estratégia militar, quando um grupo de cientistas foi convocado para decidir sobre como utilizar de maneira mais eficaz os recursos militares limitados (BELFIORE e FÁVERO, 2013). Segundo Ribeiro et al. (2018), a Pesquisa Operacional envolve "pesquisa sobre ações” e é aplicada em problemas que compreendem a condução e a coordenação das operações em uma organização.

Segundo Moreira (2013), a Pesquisa Operacional lida com problemas de como conduzir e coordenar operações em uma organização, e tem sido aplicada a diversas áreas, tais como: indústria, finanças, saúde, serviços públicos, operações militares, entre outros. 
Conforme Hillier e Lieberman (2013), uma característica a mais é que a PO tenta frequentemente encontrar uma melhor solução, conhecida como solução ótima, para o modelo que representa o problema considerado.

Segundo Arenales et al. (2015), a abordagem de resolução de um problema por meio de pesquisa Operacional envolve várias fases, tais como: definição do problema, construção do modelo, solução do modelo, validação do modelo e implementação da solução.

\subsubsection{APOIO MULTICRITÉRIO À DECISÃO}

Segundo Gomes et al. (2011), o apoio Multicritério à decisão pode ser definido como a atividade daquele, analista de decisões ou homem de estudo que, baseado em modelos claramente apresentados, mas não necessariamente formalizados, ajuda na obtenção de elementos de resposta às questões de um agente de decisão no decorrer do processo. De acordo com Almeida (2013), uma condição básica para a existência de um problema de decisão é a existência de pelo menos duas alternativas para que o decisor possa efetuar uma escolha.

Os métodos de apoio Multicritério à decisão podem ser classificados de diversas formas e uma das formas mais utilizadas é subdividi-los entre métodos da Escola Americana e métodos da Escola Francesa (MENDONÇA e RANGEL, 2017). De acordo com Bella e Costa (2016), o campo de estudo de análise multicritério ou auxílio multicritério é abordado pela literatura internacional como MCDA (Multicriteria Decision Aid) e está baseado na aplicação de métodos multicritério na solução de questões tais como: ranqueamento, classificação e seleção.

Métodos matemáticos e técnicas específicas provenientes das diversas disciplinas, como por exemplo, engenharias, sociologia, psicologia, economia e ciência política podem ser adotadas para ajudar indivíduos a realizarem DMs (Decisions Making) de qualidade (MAIA, EKEL e JÚNIOR, 2016). Segundo Silva e Rocha (2014), os métodos multicritério são categorizados em: métodos de ponderação, métodos ordinais, métodos baseados em funções de utilidade, métodos de relações de superação, métodos baseados na distância a uma alternativa ideal, entre outras.

Os métodos de apoio Multicritério à decisão têm um caráter científico e, ao mesmo tempo, apresenta capacidade de agregar todas as características consideradas importantes, inclusive as não quantitativas e esses métodos possuem foco diferenciado dos problemas e passam a atuar sob à forma de auxílio à decisão (AZEVEDO, FERREIRA e SILVA, 2016). Vale ressaltar que os métodos multicritério não apontam à uma solução ótima, mas sim direcionam para a melhor solução com base nas restrições e no perfil de preferência do decisor. (GARCEZ e FARIAS, 2016)

\subsection{MÉTODO TOPSIS}

Segundo Junior et al. (2016), o método TOPSIS (Technique for Order Preference by Similarity to na Ideal Solution) proposto por Hwang e Yoon (1981), vem sendo aplicado a pesquisas científicas voltadas ao apoio a decisão multicritério. De acordo com Medeiros et al. (2017), o método TOPSIS posiciona as alternativas de acordo com sua distância da solução ideal positiva (PIS) e da solução ideal negativa (NIS).

Segundo Oliveira et al. (2018), para a aplicação do TOPSIS, uma série de cálculos deve ser executada:

- Preliminares (objetivos, matriz, pesos e impactos);

- Normalização dos dados;

- Ponderação dos dados; 
- Determinação da solução ideal (PIS) e anti-ideal (NIS);

- Cálculo das distâncias D+ e D- para cada alternativa;

- Cálculo dos coeficientes C para cada alternativa;

- Ordenação das alternativas por coeficientes. seguir.

As equações ou a modelagem matemática do método podem ser observadas a

$$
\sum_{i=1}^{n} w=1
$$

Com a equação (1), o decisor atribui pesos aos critérios e esses pesos somados não podem ultrapassar o valor 1 , ou seja, $100 \%$.

$$
n_{i j}=\frac{x_{i j}}{\sqrt{\sum_{j=1}^{n} x_{i j}^{2}}}, i=1,2 \ldots, m ; j=1,2, \ldots, n .
$$

Com a equação (2), o decisor pode normalizar a matriz de decisão com os critérios elencados por ele.

$$
\boldsymbol{p}=\left[p_{i j}\right] \boldsymbol{m} x \boldsymbol{n}: p_{i j}=w_{j} x r_{i j}
$$

Utilizando a equação (3), o decisor pode multiplicar os valores da matriz de decisão normalizada pelos pesos impostos por ele.

$$
\begin{aligned}
& \boldsymbol{d}_{i}^{+}=\sqrt{\sum_{j=1}^{n}\left(\boldsymbol{d}_{i j}\right)^{2}} \rightarrow \boldsymbol{p}_{j}^{+}-\boldsymbol{p}_{i j} \\
& \boldsymbol{d}_{i}^{-}=\sqrt{\sum_{j=1}^{n}\left(\boldsymbol{d}_{i j}\right)^{2}} \rightarrow \boldsymbol{p}_{j}^{-}-\boldsymbol{p}_{i j}
\end{aligned}
$$

Com as equações (4) e (5), o decisor calcula as distâncias Euclidianas para o vetor de soluções positivas A+ e para o vetor de soluções negativas A-. Onde $\boldsymbol{p}_{j}^{+}-\boldsymbol{p}_{i j}$ é a solução ideal positiva para critérios de benefício e $\boldsymbol{p}_{j}^{-}-\boldsymbol{p}_{i j}$ é a solução ideal negativa para critérios de custo.

$$
R C_{i}=\frac{S_{j}^{-}}{S_{j}^{+}+S_{j}^{-}}
$$

\section{ESTUDO DE CASO}

Como mencionado anteriormente, o presente estudo de caso tem como propósito selecionar o melhor congresso para um aluno de graduação em Engenharia de Produção publicar um artigo. Para alcançar tal feito, foram feitas entrevistas com cinco professores especialistas na área e foram elencados as seguintes alternativas e os seguintes critérios como mostra o Quadro 1 a seguir.

Quadro 1: Alternativas e critérios do estudo 


\begin{tabular}{|l|l|}
\hline \multicolumn{1}{|c|}{ Alternativas } & \multicolumn{1}{c|}{ Critérios } \\
\hline $\mathrm{A}_{1}$ - SIMPEP & $\mathrm{C}_{1}$ - Importância \\
\hline $\mathrm{A}_{2}$ - SIMEP & $\mathrm{C}_{2}$ - Custos Envolvidos \\
\hline $\mathrm{A}_{3}$ - ENEGEP & $\mathrm{C}_{3}$ - Nivel dos Artigos \\
\hline $\mathrm{A}_{4}$ - SBPO & $\mathrm{C}_{4}$ - Conveniência das Datas \\
\hline $\mathrm{A}_{5}$ - CNEG & $\mathrm{C}_{5}$ - Facilidade de Acesso \\
\hline $\mathrm{A}_{6}$ SEGET & $\mathrm{C}_{6}$ - Qualidade do site \\
\hline
\end{tabular}

Fonte: Autores (2019)

Vale ressaltar que foram escolhidos os congressos que há maior procura para a publicação de artigos e foram elencados os dois mais importantes na área de engenharia de produção nacional como o SBPO (Simpósio Brasileiro de Pesquisa Operacional) e o ENEGEP (Encontro Nacional de Engenharia de Produção).

Feito esse processo, foram atribuídas as notas para os critérios de: importância, nível dos artigos, conveniência das datas, facilidade de acesso e qualidade do site. Para a atribuição dessas notas, foi usada uma escala Likert de 6 pontos, onde 1 é a pior nota e 6 é a melhor. Para a atribuição dos custos envolvidos, como já mencionado em tópico anterior, foram feitas pesquisas na internet e no site do decolar.com para os seguintes itens: inscrição no evento e taxa de publicação, passagem para o local do evento, hospedagem. Para as refeições foi sugerido um valor de $\mathrm{R} \$ 200,00$ para os três dias e no que diz respeito à locomoção foi sugerido um valor de $\mathrm{R} \$ 150,00$ para os três dias do evento.

Para o evento do SIMPEP (Simpósio de Engenharia de Produção), os custos foram os seguintes: inscrição e taxa de publicação resultando em valor total de $\mathrm{R} \$ 535,00$, passagem de ônibus resultando em um valor de aproximadamente $\mathrm{R} \$ 218,99$ e hospedagem para três dias com um valor de $\mathrm{R} \$ 330,00$, além de refeições e locomoção já mencionados antes chegando a um valor total de aproximadamente R $\$ 1.433,99$. Para o evento do SIMEP (Simpósio de Engenharia de Produção) os valores foram de: inscrição e taxa de publicação em um valor de $\mathrm{R} \$ 145,00$, passagem de avião em um valor de $\mathrm{R} \$ 371,00$, hospedagem para três dias resultando em um valor de $\mathrm{R} \$ 473,00$ além de refeições e locomoção resultando em valor total de aproximadamente $\mathrm{R} \$ 1.339,00$.

Para o evento do ENEGEP (Encontro Nacional de Engenharia de Produção), os custos foram de: inscrição e taxa de publicação de $\mathrm{R} \$ 450,00$, passagem de ônibus de aproximadamente $\mathrm{R} \$ 231,10$, hospedagem em um valor de $\mathrm{R} \$ 552,00$ além de refeições e locomoção resultando em um valor total de aproximadamente $\mathrm{R} \$ 1.583,10$. Para o evento do SBPO (Simpósio Brasileiro de Pesquisa Operacional) os custos para a inscrição e taxa de publicação em um valor de $\mathrm{R} \$ 200,00$, a passagem de ônibus em um valor de aproximadamente $\mathrm{R} \$ 274.24$, hospedagem em um valor de $\mathrm{R} \$ 329,00$ além do deslocamento e das refeições, resultando em um valor de aproximadamente R\$1.153,24.

Para o CNEG (Congresso Nacional de Excelência em Gestão), o processo foi um pouco diferente, pois esse evento ocorre na cidade do Rio de Janeiro e este trabalho tem como um dos autores um estudante que mora na cidade e então os custos envolvidos foram: inscrição e taxa de publicação em um valor de $\mathrm{R} \$ 260,00$ e nesse caso foi estipulado um valor de $\mathrm{R} \$ 100,00$ para o deslocamento e o mesmo valor para as refeições nos dois dias do evento, resultando em um valor de $\mathrm{R} \$ 460,00$. Para o SEGET (Simpósio de Excelência em Gestão e Tecnologia), os custos foram: inscrição e taxa de publicação em um valor de $\mathrm{R} \$ 490,00$, passagem de ônibus no valor de aproximadamente $\mathrm{R} \$ 55,91$, hospedagem em um valor de $\mathrm{R} \$ 541,00$ para os três dias de evento além do deslocamento e refeições iguais aos demais eventos excluindo o CNEG, resultando em um valor de aproximadamente $\mathrm{R} \$ 1.436,91$. 
Explicitados todos esses valores, foi aplicado o método TOPSIS a fim de ranquear os eventos e ver qual é o melhor para a publicação de um artigo científico mediante o cenário proposto e as opiniões dos profissionais.

\subsection{APLICAÇÃO DO MÉTODO TOPSIS}

Como já mencionado antes, foram atribuídos os valores para os critérios a fim de montar a matriz de decisão do problema, a qual pode ser observada no Quadro 2 a seguir.

Quadro 2: Matriz de Decisão do problema estudado

\begin{tabular}{|c|c|c|c|c|c|c|}
\hline An / Cn & Custos & Importância & $\begin{array}{c}\text { Nível dos } \\
\text { artigos }\end{array}$ & $\begin{array}{c}\text { Qualidade } \\
\text { do site }\end{array}$ & Datas & Acesso \\
\hline SIMPEP & $1.433,99$ & 4 & 4 & 6 & 1 & 2 \\
\hline SIMEP & $1.339,00$ & 3 & 3 & 1 & 6 & 1 \\
\hline ENEGEP & $1.583,10$ & 5 & 5 & 5 & 2 & 4 \\
\hline SBPO & $1.153,24$ & 6 & 6 & 4 & 5 & 3 \\
\hline CNEG & 460,00 & 1 & 1 & 3 & 4 & 6 \\
\hline SEGET & $1.436,91$ & 2 & 2 & 2 & 3 & 5 \\
\hline
\end{tabular}

Fonte: Autores (2019)

A partir desta matriz de decisão, foi feita a normalização da mesma com a equação (2) já mencionada em tópico anterior. A matriz de decisão normalizada pode ser observada no Quadro 3 a seguir.

Quadro 3: Matriz de Decisão normalizada

\begin{tabular}{|c|c|c|c|c|c|c|}
\hline An / Cn & Custos & Importância & $\begin{array}{c}\text { Nível dos } \\
\text { artigos }\end{array}$ & $\begin{array}{c}\text { Qualidade do } \\
\text { site }\end{array}$ & Datas & Acesso \\
\hline SIMPEP & 0,454338 & 0,4193139 & 0,419313935 & 0,628970902 & 0,10483 & 0,20966 \\
\hline SIMEP & 0,424242 & 0,3144855 & 0,314485451 & 0,104828484 & 0,62897 & 0,10483 \\
\hline ENEGEP & 0,501581 & 0,5241424 & 0,524142418 & 0,524142418 & 0,20966 & 0,41931 \\
\hline SPBO & 0,365386 & 0,6289709 & 0,628970902 & 0,419313935 & 0,52414 & 0,31449 \\
\hline CNEG & 0,145744 & 0,1048285 & 0,104828484 & 0,314485451 & 0,41931 & 0,62897 \\
\hline SEGET & 0,455263 & 0,209657 & 0,209656967 & 0,209656967 & 0,31449 & 0,52414 \\
\hline Peso & 0,35 & 0,25 & 0,20 & 0,05 & 0,05 & 0,10 \\
\hline
\end{tabular}

Fonte: Autores (2019)

Com a matriz de decisão normalizada, foram atribuídos os pesos para cada critério como foi visto no Quadro 3. Esses pesos foram atribuídos a partir de entrevistas feitas com os dois pesquisadores na área. Feito esse processo, foi feita a multiplicação da matriz de decisão normalizada pelos pesos atribuídos utilizando a equação (3) mencionada em tópico anterior, o qual pode ser observado no Quadro 4 a seguir. 


\begin{tabular}{|c|c|c|c|c|c|c|}
\hline An / Cn & Custos & Importância & $\begin{array}{c}\text { Nível dos } \\
\text { artigos }\end{array}$ & $\begin{array}{c}\text { Qualidade do } \\
\text { site }\end{array}$ & Datas & Acesso \\
\hline SIMPEP & 0,159018 & 0,1048285 & 0,083862787 & 0,031448545 & 0,00524 & 0,02097 \\
\hline SIMEP & 0,148485 & 0,0786214 & 0,062897090 & 0,005241424 & 0,03145 & 0,01048 \\
\hline ENEGEP & 0,175553 & 0,1310356 & 0,104828484 & 0,026207121 & 0,01048 & 0,04193 \\
\hline SBPO & 0,127885 & 0,1572427 & 0,125794180 & 0,020965697 & 0,02621 & 0,03145 \\
\hline CNEG & 0,051010 & 0,0262071 & 0,020965697 & 0,015724273 & 0,02097 & 0,06290 \\
\hline SEGET & 0,159342 & 0,0524142 & 0,041931393 & 0,010482848 & 0,01572 & 0,05241 \\
\hline
\end{tabular}

Fonte: Autores (2019)

A partir da matriz de decisão normalizada multiplicada pelos pesos atribuídos, foram encontradas a solução ideal (A+) e a solução anti-ideal (A-), os quais podem ser observadas no Quadro 5 a seguir.

Quadro 5: Solução ideal e solução anti-ideal

\begin{tabular}{|c|c|c|c|c|c|c|}
\hline A+ & 0,051010 & 0,1572243 & 0,12579418 & 0,031448545 & 0,03145 & 0,06290 \\
\hline A- & 0,175553 & 0,2620710 & 0,02965697 & 0,005241424 & 0,00524 & 0,01048 \\
\hline
\end{tabular}

Fonte: Autores (2019)

Com a posse da solução ideal e da solução anti-ideal, foram calculadas as distâncias relativas às soluções ideal e anti-ideal utilizando as equações (4) e (5) já mencionadas antes. Feito esse processo, foi calculada a proximidade relativa em relação a $A_{+}$e A- utilizando a equação (6). Estes passos podem ser observados no Quadro 6 a seguir.

Quadro 6: Distância relativa e proximidade relativa

\begin{tabular}{|c|c|c|c|}
\hline An / Cn & Di+ & Di- & RCi \\
\hline SIMPEP & 0,13553 & 0,169591 & 0,5558228 \\
\hline SIMEP & 0,15189 & 0,248396 & 0,6205487 \\
\hline ENEGEP & 0,13338 & 0,155723 & 0,5386410 \\
\hline SBPO & 0,08388 & 0,153721 & 0,6469642 \\
\hline CNEG & 0,16885 & 0,272622 & 0,6175243 \\
\hline SEGET & 0,17479 & 0,215094 & 0,5516903 \\
\hline
\end{tabular}

Fonte: Autores (2019)

Após a obtenção da distância relativa em relação à solução ideal e à solução antiideal, chegou-se ao seguinte ranking que pode ser observado no Quadro 7 a seguir.

Quadro 7: Classificação dos congressos 


\begin{tabular}{|c|c|c|}
\hline Classificação & Congresso & $\mathrm{RCi}$ \\
\hline $1^{\circ}$ & SBPO & 0,646964 \\
\hline $2^{\circ}$ & SIMEP & 0,620549 \\
\hline $3^{\circ}$ & CNEG & 0,617524 \\
\hline $4^{\circ}$ & SIMPEP & 0,555823 \\
\hline $5^{\circ}$ & SEGET & 0,55169 \\
\hline $6^{\circ}$ & ENEGEP & 0,538641 \\
\hline
\end{tabular}

Fonte: Autores (2019)

\section{DISCUSSÃO DOS RESULTADOS}

Após a utilização do método TOPSIS, o resultado da proximidade relativa mostrou a ordem dos congressos e viu-se que o mais apropriado evento para se publicar um artigo é o SBPO, levando em conta as opiniões dos cinco professores que foram entrevistados. Esse resultado pode mudar, pois a opinião de cada profissional varia muito e este pode dar notas e pesos diferentes dos que foram atribuídos neste estudo de caso mudando assim o resultado. Pode-se dizer que o congresso SBPO foi o primeiro colocado, pois as notas dele são as melhores, levando apenas uma nota baixa de 3 pontos no critério de acesso. Este evento é o segundo com menor custo perdendo apenas para o CNEG. Vale ressaltar que o ranking ficou bastante apertado, pois no resultado final o SBPO foi seguido de perto pelo SIMEP, que não teve as melhores notas, tendo apenas uma nota excelente no critério de conveniência das datas. Em seguida veio o CNEG bem próximo ao SIMEP, podendo ser dito que ele ficou com a $3^{\mathrm{a}}$ posição, pois tem o menor custo dentre todos e ficou com apenas uma nota excelente no critério de facilidade de acesso. Ficando com o $4^{\circ}$ lugar veio o SIMPEP com proximidade relativa de 0,555823 , ganhando esta posição, pois suas notas foram razoáveis tendo uma noto máxima em qualidade do site e tendo a pior nota em conveniência das datas. Vale ressaltar também que o SIMPEP é o terceiro evento com maior custo ficando atrás do ENEGEP e do SEGET. Logo após o SIMPEP veio o SEGET com proximidade relativa de 0,55169, podendo ressaltar que é o segundo evento com maior custo e que suas notas não foi as melhores, tendo apenas uma nota alta no critério de facilidade de acesso. E por último veio o ENEGEP o evento com maior custo e com três notas altas nos critérios de importância, nível doas artigos e qualidade do site tendo nos outros critérios notas razoáveis.

Vale ressaltar que o método TOPSIS é compensatório, ou seja, mesmo o evento não tendo as melhores notas, no decorrer dos cálculos o algoritmo compensa essa perda fazendo com que o congresso fique ao final dos cálculos com uma boa posição como foi o caso do CNEG que ficou com a terceira colocação tendo as piores notas dentre todos os outros eventos.

\section{CONSIDERAÇÕES FINAIS}

O método TOPSIS é um modelo multicritério que pode ser utilizado em qualquer tipo de problema, podendo ser mais interessante naquele problema em que há várias alternativas e vários critérios. Foi utilizado este método no presente artigo, pois como mencionado antes, ele é um método compensatório durante a execução de seus cálculos.

Feito todo o processo matemático, foi visto que o evento do SBPO ficou com o primeiro lugar com uma proximidade relativa de 0,646964, seguido do SIMEP, CNEG, SIMPEP, SEGET e ENEGEP. O resultado da pesquisa mostrou-se satisfatório com a realidade criada com base nas opiniões dos profissionais além disso o resultado foi discutido 
com eles e como mencionado antes, o resultado pode variar com as mais diversas opiniões dos profissionais da área de Engenharia de Produção.

\section{REFERÊNCIAS}

ALMEIDA, A. T. Processo de decisão nas organizações: construindo modelos de decisão multicritério. São Paulo: Atlas, 2013.

ARENALES, M., et al. Pesquisa Operacional para cursos de engenharia. 2.ed. Rio de Janeiro: Elsevier, 2015.

AZEVEDO, T. N., FERREIRA, M. M. G., SILVA, R. G. A utilização dos métodos de apoio multicritério à decisão no Brasil, entre os anos de 2007 a 2017: um estudo bibliométrico. Anais XXXVIII Encontro Nacional de Engenharia de Produção. Maceió/AL, 2016.

BELLA, R. L. F., COSTA, H. G. Auxílio multicritério na priorização de riscos de projetos. Anais XLVIII Simpósio Brasileiro de Pesquisa Operacional. Vitória/ES, 2016.

BELFIORE, P., FÁVERO, L. P. Pesquisa Operacional para cursos de engenharia. Rio de Janeiro: Elsiever, 2013.

GARCEZ, T. V., FARIAS, D. Priorização dos equipamentos através da metodologia multicritério de apoio à decisão alinhada com a visão estratégica do WCM. Anais XLVIII Simpósio Brasileiro de Pesquisa Operacional. Vitória/ES, 2016.

GOMES, L. F. A. M., ARAYA, M. C. G., CARIGNANO, C. Tomada de decisões em cenários complexos: introdução aos métodos discretos do apoio multicritério à decisão. São Paulo: Cengage Learning, 2011.

HILLIER, F. S., LIEBERMAN, G. J. Introdução à pesquisa operacional. 9. ed. Porto Alegre: AMGH Ed.; McGraw-Hill, 2013.

JUNIOR, M. M. R., KREUZBERG, F., JESKE, M., RODIRGUES, F. Métodos TOPSIS e VIKOR na verificação da resource advantage theory em empresas têxteis brasileiras. Anais XVIII Simpósio de Pesquisa Operacional e Logística da Marinha. Rio de Janeiro/RJ, 2016.

MAIA, W. F. S., EKEL, P. I., JÚNIOR, P. P. C. Avaliação de riscos de subestações para a prevenção de acidentes: análise de fatores contribuintes. Anais XLVIII Simpósio Brasileiro de Pesquisa Operacional. Vitória/ES, 2016.

MEDEIROS, M., DANTAS, A. S., DANTAS, M. R., FERREIRA, L. Classificação de desempenho de franqueados utilizando o TOPSIS-SORT. Anais XLIX Simpósio Brasileiro de Pesquisa Operacional. Blumenau/SC, 2017.

MENDONÇA, T. C., RANGEL, L. A. D. Priorização de equipamentos em manutenção empregando o apoio Multicritério à decisão. Anais XLIX Simpósio Brasileiro de Pesquisa Operacional. Blumenau/SC, 2017.

MOREIRA, D. A. Pesquisa Operacional: curso introdutório. 2. Ed. São Paulo: Thomson Learning, 2013. 
OLIVEIRA, A. S., GOMES, C. F. S., BARROS, M., FERREIRA, G. S., BARCELOS, M. R. S. Ordenação de critérios para avaliação de propostas de incubação de empresas: uma abordagem multicritério. Anais XXXVIII Encontro Nacional de Engenharia de Produção. Maceió/AL, 2018.

RIBEIRO, J., MORAES, A., SILVA, J. Q. B., VASCONCELOS, C. R. P. Análise do comportamento do fluxo de atendimento do serviço de almoço de um restaurante universitário utilizando o modelo de teorias das filas. Anais XXXVIII Encontro Nacional de Engenharia de Produção. Maceió/AL, 2018.

SILVA, G. B., ROCHA, P. M. Análise experimental multicritério de auxílio à tomada de decisão na seleção de pessoal para um projeto desenvolvimento de software. Anais XVII Simpósio de Pesquisa Operacional e Logística da Marinha. Rio de Janeiro/RJ, 2014. 